\title{
A REVIEW OF FAULT DIAGNOSISIN MECHATRONICS SYSTEMS
}

\author{
Daniel Cordoneanu ${ }^{1}$ and Constantin Niţu ${ }^{2}$ \\ 1,2 University "Politehnica" of Bucharest-Department of Mechatronics and Precision Mechanics \\ 313, Splaiul Independentei, Bucharest 060042, Romania \\ E-mail: daniel.cordoneanu@stud.mec.upb.ro
}

\begin{abstract}
Fault diagnosis gets increasing importance in today's production environment and with the advancements in the field of artificial intelligence, researchers look for new ways to keep a system away from faults, that would interrupt the production. In recent years, many papers were written regarding this subject especially regarding predictive maintenance and fault diagnosis. This paper presents recent works that expose new methods for intelligent fault diagnosis. This step is important for future research in order to have a better understanding of state of the art algorithms and look for ways to improve the existing fault diagnosis approaches. The focus will be on electrical systems and actuators and manipulating systems (robots), production experience showing that the mechanical parts are the most exposed to production-ending faults. That's why most of the observer systems are using vibrations as the main data for their algorithms, but also other measurements can provide useful information about the condition of a system.
\end{abstract}

Keywords: Fault Diagnosis, Predictive Maintenance, Mechatronics Systems, Artificial Intelligence

\section{Introduction}

Mechatronics systems cover a wide area in today's production machines. Mechanical parts and electronics are fused together and now, with the Internet of Things (IOT), intelligent control algorithms can operate and control the machines remotely, having all the necessary power of computing at their disposal. However, we can split the mechatronics systems into multiple types:

- actuators - electrical motors, pneumatic and hydraulic motors, etc.

- mechanical drives - gearboxes, belt pulleys, worm gears, etc.

- sensors and data acquisition systems - force sensors, accelerometers, temperature sensors, etc.

Further, methods of condition monitoring and fault diagnosis for each of these subsystems will be presented, and then review ways of monitoring them as a whole, in complex systems.

The methods are often divided into multiple categories, as Mouzakitis describes the fault diagnosis systems in [1]:

- model-based methods

- data-based methods

- hardware-based methods

Further it will be presented different combinations of the methods used for the systems mentioned before. Firstly, a better differentiation between these methods is graphically shown. In figure 1,there are presented some of the algorithms that are used to implement the above-mentioned methods. These algorithms are well described by Mouzakitis in his article [1] and some of them will be further discussed in this paper, based on fault diagnosis examples for certain systems.

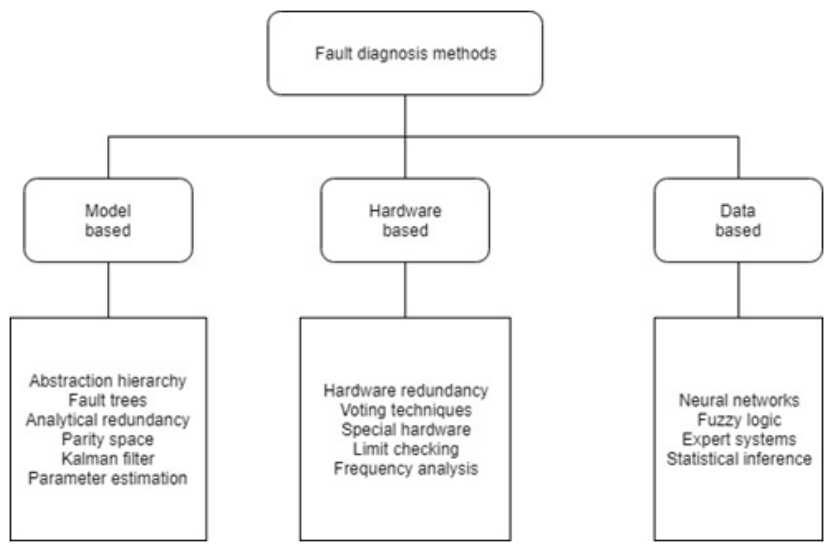

Figure 1: Fault diagnosis methods and algorithms for implementation 
Figures 2 and 3 show the main difference between model-based and data-based methods.

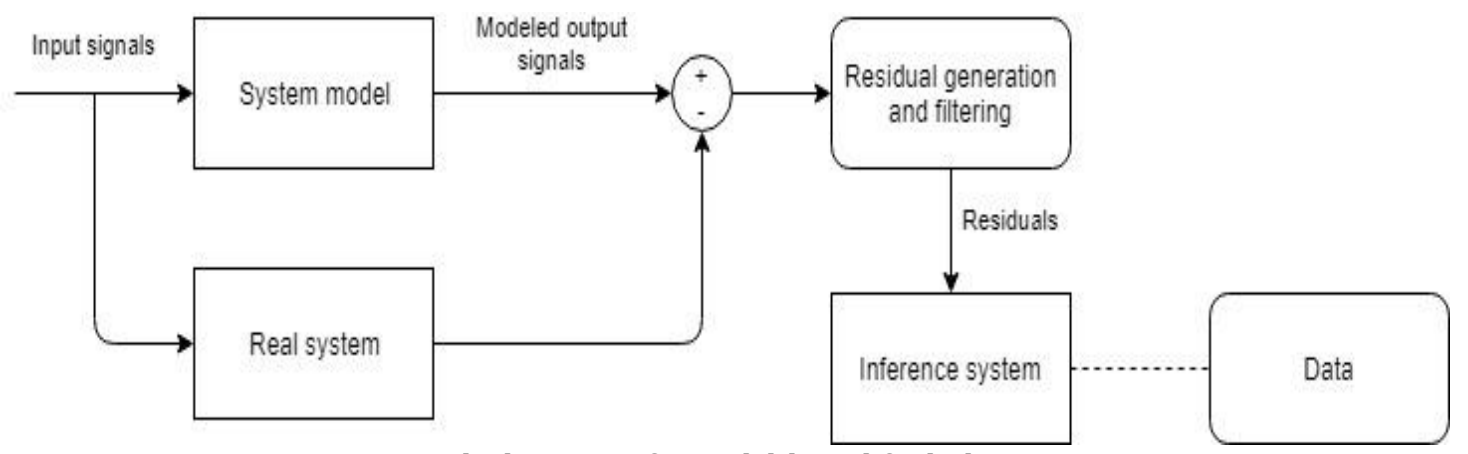

Figure 2: Simple diagram of a model-based fault diagnosis system

As it can be seen in the two figures, they are pretty much the same at a macro system level, but the main difference consists of how the residual information is generated. The model-based one needs no prior data to get an expected result, while the data-based system requires information on which an internal model of the system is built. The quality and quantity of the training data is very important (as it is for most machine learning algorithms) and also the system has to be properly designed, in order to avoid the training data over fit or under fit.

In both cases, the expected output would be erroneous, leading to a bad fault diagnosis. On the other hand, if the model-based system is too simple and does not capture all the dynamics of the system, then the fault diagnosis system may not be able to detect all the faults that may appear. In both cases, the inference system can be an artificial intelligence system, which needs data to be trained as well. In data-based methods, the statistical method scan also be included. For example, particle filters algorithms are very well described in [2] by Verma et al. They put in balance the computational power that is needed in a simple model-based fault diagnosis method and in a stochastic method. The latter, although not being as accurate as the model-based method, is more efficient and takes less time to process the signals and extract residual features needed for the health monitoring algorithms.

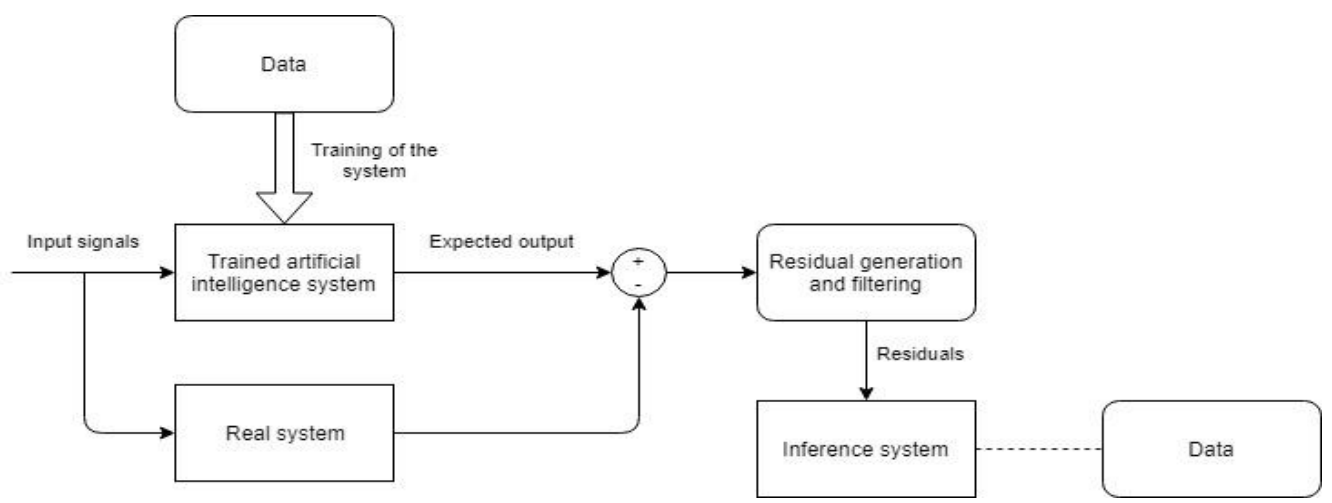

Figure 2: Simple diagram of a data-based fault diagnosis system

An architecture example for fault diagnosis systems is given by Catterson and McArthur in [3], where a Multi-Agent system for the condition monitoring of a power transformer is presented. This one is organized by layers, that should be part of any fault diagnosis system, as COMMAS architecture, where they mention that each system agent can be represented by a different algorithms (a modular approach). These layers are, in fact, the following 4 tasks:

- data monitoring

- interpretation layer

- corroboration layer

- information layer

\section{Fault Diagnosis for Actuating Systems}

The actuating systems play a very important role in every dynamic system, because they are the "fuel" of the production systems.

Starting with a model-based fault diagnosis approach, Rolf Isermann presents some faultdetection methods based on the system model and then points out an application of these ones on a real system [4]. The methods that can be used are summarized as:

- process models and fault modelling

- fault-detection with parameter estimation

- fault-detection with observers

- fault-detection with signal models 
The author's diagram of a model-based fault detection system is shown in figure 4:

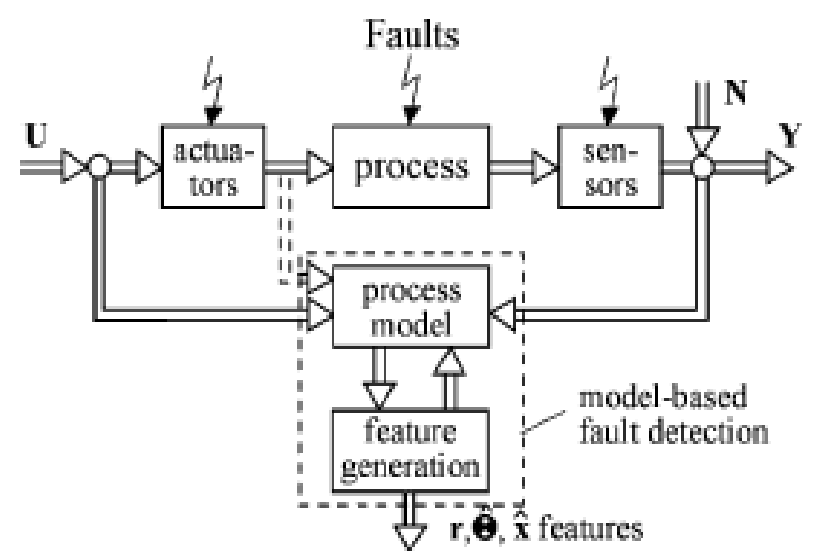

Figure 4: Scheme of process model-based fault-detection [4]

In the article, the author presents an example of a fault diagnosis algorithm for a cabin pressure outflow valve actuator.

It is not only an interesting case, but also safety related since the cabin pressure is vital for the passengers. There are two brushless direct current (BLDC) motors, one of them being a backup, if the other one has a fault.

The fault-detection method is a combination of parameter estimation and parity equations. Having a detailed BLDC motor model for all three phases and based on the model as well as the available measurements, the needed parameters for generating residuals were estimated and the parity equations were created.

The measured values are control voltage - $\mathrm{U}(\mathrm{t})$, armature current-I(t), the angular position of the shaft and of the flap. The last two are related by a gear ratio. The residuals allow a monitoring algorithm to detect and isolate the faults (in this case, a fuzzy logic system). Each residual represent a certain measure and based on these signals, the system can detect the part of the system that is faulty.

The author's model is presented in figure 5.

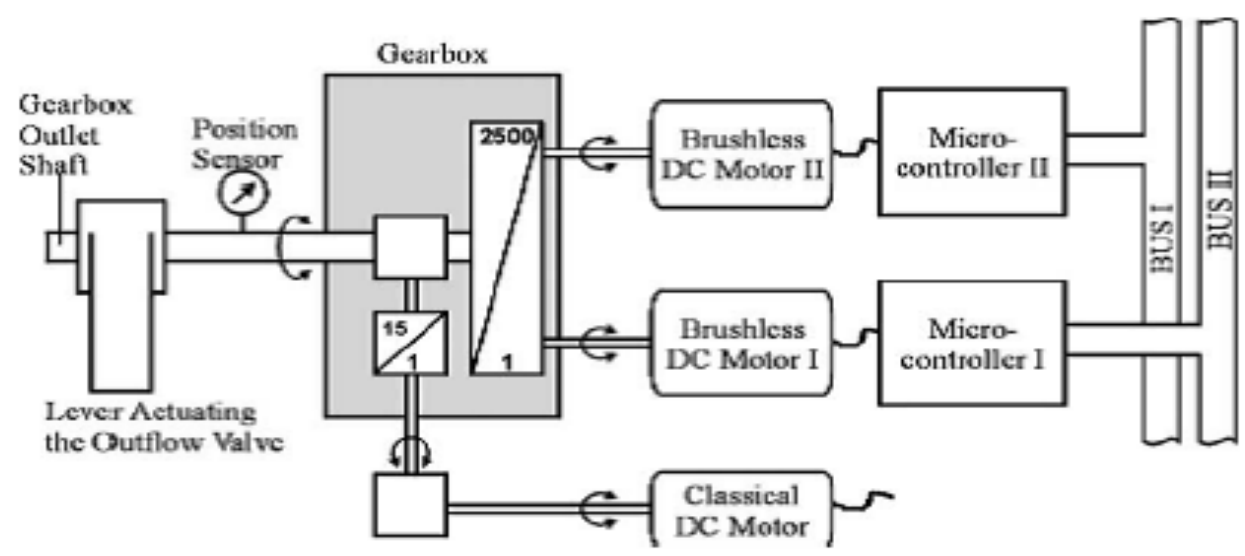

Figure 5: Redundant DC motor drive system for the outflow valve [4]

In this example, it can be noticed that the electricity provides the information about the state of the system.

A model-based solution gives the advantage that each part of the system is described and faults isolation is only a matter of comparing the modeled signal with the real one, thus obtaining residuals which can further be stored in a fault matrix.

One disadvantage of this method is encountered when the system has non-linear dynamics and multiple components and consists of a lot of computational power consuming.
Another drawback is the need for changing the model, if a part of the system is replaced with a different part which has the same output, but different behavior.

Another model-based fault diagnosis method is proposed by Cho et al [5]. Their method is based on a stochastic model using only the current measured by a Hall sensor.

The authors' algorithm is best described by the diagram in figure 6.

The main advantage of this approach is that the probabilistic model is part of the system and the 
inputs that go into the real system do not need to be computed again through the system's model.
Therefore this kind of method is very efficient, having a low computational load.

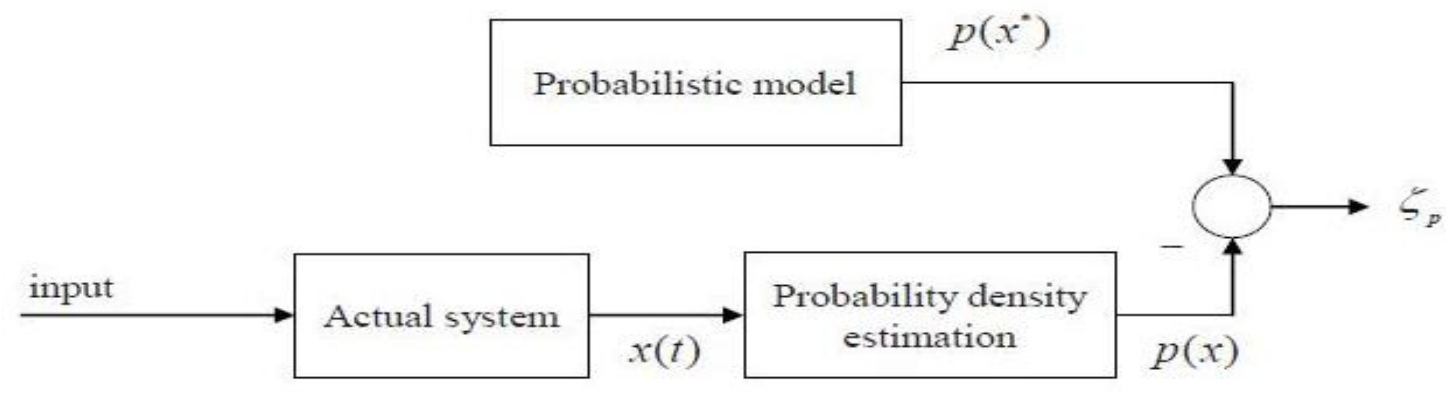

Figure 6: Stochastic model based fault detection scheme [5]

To address a general situation where a model is hard to create, data-based solutions are the best fit.

For instance, in [6], Hua Su and KilTo Chang propose a fault diagnosis method based on vibration signal and on vibration residuals created by a neural network. It can be seen in figure 7 the model that the schematic proposed by the authors is more or less the same with the one illustrated in figure 3.

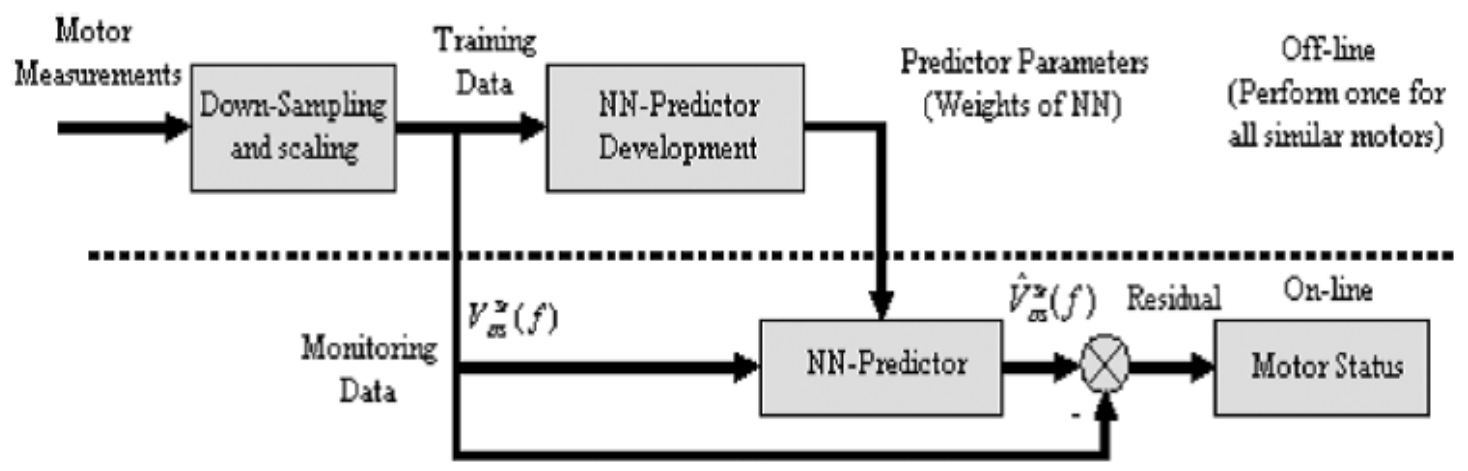

Figure 7: Principle of analytical redundancy method using neural network modelling [6]

The system which is monitored is an induction machine, a widely spread electric actuator in the industry. An induction machine's model has many non-linear characteristics and to create a dynamic model of this system is time consuming and also takes a lot of computing power. Temperature, inverter variations, etc. are all factors which come into play when analyzing the vibrations signals that come from this type of machine. As the authors mention, vibrations signals are accepted as a good indicators of the stator, rotor and bearings condition.

Comparing this method with the first one, some differences have to be mentioned:

- model-based methods give a more clear indication about the source of the faults

- data-based methods are more versatile because they can capture non-linear dynamics without having to know specific parameters of the system

- both methods use in the end residuals as inference parameters on whether there is a fault in the system or not

An alternative for dynamic system efficient monitoring is based on vibration analysis. In [7] the authors state that "vibration analysis techniques are more effective in assessing a machine's health". In this paper, a neural network is used to create a databased model for the electrical behavior of the machine.

The data used is extracted from the machine vibration signals and they represent quasi-steady signals used to remove unwanted disturbances.

Further, the neural network output is then used to create the analytical residuals on which the fault diagnosis inference is based on. Both in [6] and [7], this type of fault diagnosis using artificial intelligence is up to the task of modeling properly a non-linear dynamic system and the output can be used then to assess the system's health condition.

\section{Fault Diagnosis for Mechanical Drives}

The motion transmission systems play an important role in every engineering system because they are the ones which transfer the power developed by the actuating systems.

That's why fault diagnosis techniques have been developed for these systems as well. A challenge, however, for this type of systems is to create a general fault diagnosis system, which is not aimed to a certain type of components, but is focused on the 
kinetic energy transfer chain: actuator - mechanical drive - moving object. For example, the same type of motion can be achieved using a gear-rack transmission or a screw drive: they both convert a rotational motion to a linear one.

For example, in [8], Wang and Wong propose a model-based fault diagnosis technique for gear faults that relies on signal averaging technique. A model is firstly created, based on the vibration of the monitored gear in its healthy state.
This model is then used as an error filter to compute the future-state of the vibration signal from the same gear. The authors' algorithm is presented in figure 8.

As the diagram stops at the prediction error, the algorithm can be improved by using this error as data for a machine learning technique that would give more insight into what type of fault it is and, maybe, having the gears still working at a lower speed (having a fault tolerance).

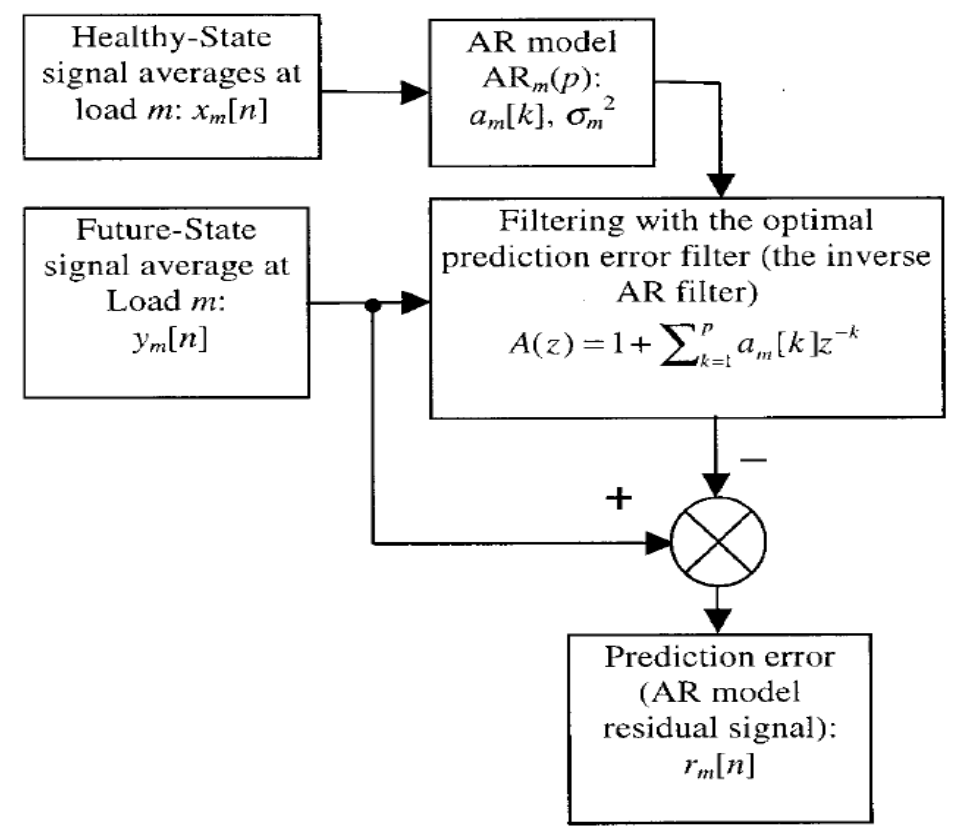

Figure 8: Model-based fault diagnosis of a gear [8]

The previously described fault diagnosis methods were applied to a belt conveyor, in order to make a comparison of results. In [9], Ghoshal and Samanta present a method in which the belt conveyor system is observed and diagnosed using a model-based approach. This one makes use of bond graphs formalism to model the system, which is based on the power exchange between the ideal components of the dynamical systems. Mainly, there are power variables sources, transformers, storing and dissipative elements, characterized by lumped parameters. Moreover, this way of modeling is independent on physical domain, enabling unitary representation of the multidisciplinary systems. For example, the belt conveyor model, shown in figure 9, involves both electrical and mechanical domain.

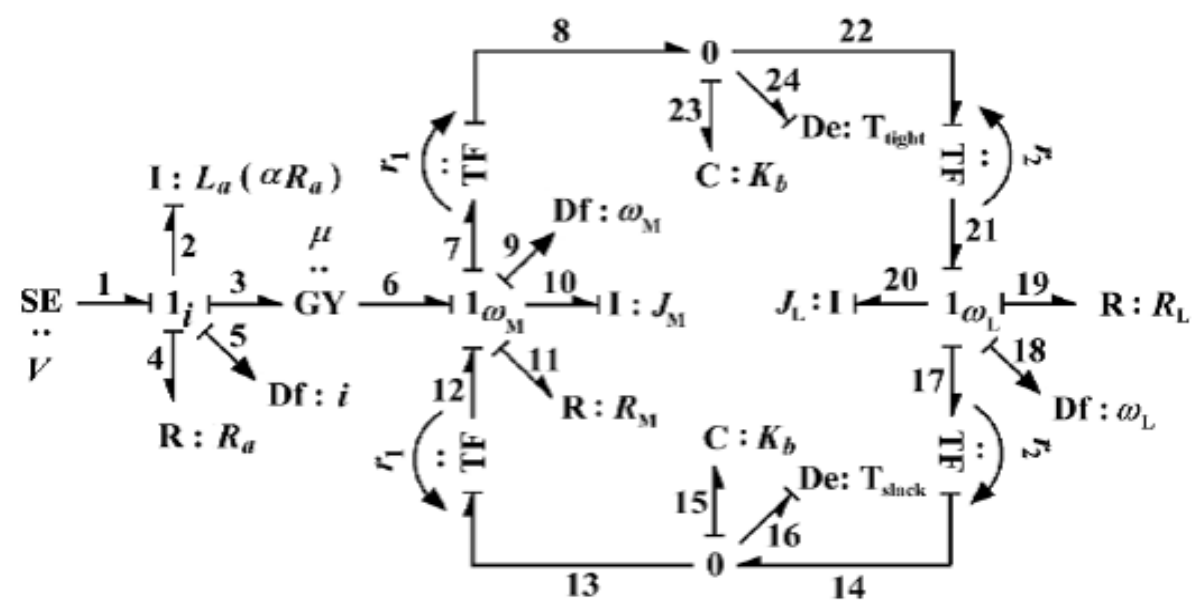

Figure 9: Bond-graph model for a belt conveyor system [9] 
Using this type of modeling, the authors have relied on Analytical Redundancy Relations (ARR), which are constraint relationships between inputs, outputs and known process parameters (or estimated). The numerical result of ARR called residual is then used to asses if there is a fault present or not in the system. Also, these residuals are used to isolate the faults by help of a fault signature matrix. Because in real time applications the residuals which result after processing the data from the sensors will never be zero (sensor noises, parameters uncertainties), a coherence vector is built for each residual that takes into account some threshold values. A fault is detected when one of the elements of the coherence vector is different to zero.

Having the ARRs in differential form (so someone can look backwards in time to see what happened) and getting the residuals in comparison with the fault signature matrix, it is possible to identify which element of the bond graph is responsible for the fault. This way, the corresponding real system component is identified. In this example, a simple belt drive with two pulleys is modeled and simulations are run for the cases when there are no faults and when there are faults. The losses due to damping or jamming of the pulley are measured in the energy-dissipative elements of type $\mathrm{R}$ (the damping coefficients of the two pulleys), which will further be observed and based on their values, assertions of different faults can be made. The sensors measure the motor current, the rotational speeds of the pulleys and the tensile forces in the two branches of the belt. This type of fault diagnosis is pretty straight forward and easy to implement, the hardest part of it being the modeling one and finding out the correct threshold values for the coherence vector.

In [10], Li et al. analyze a system that has a belt drive, this time the fault diagnosis method involving an artificial intelligence system similar to neural networks - support vector machine (SVM). The only collected data is from vibration in order to have the smallest number of sensors. This data is further processed to retrieve the wavelet packets (as the authors point out, this type of data is efficient for representing a rotary machine). The wavelet package decomposition (WPD) is based on wavelet analysis, giving an improved time-frequency resolution. Once the signal was filtered and the energy for each frequency band was obtained, this data can be used as input to the SVM system.

The SVM is an algorithm based on statistical methods that copes very well with nonlinear, finitesample, high-dimensionality problems. This algorithm is efficiently used to classify the state of the conveyor belt and idlers. In this specific scenario, the authors point out that in real applications it's pointless to add sensors to each idler (and not costefficient), so they added accelerometers once every three idlers. The data that come from these sensors are then filtered out to WPD and represent the input of the SVM system. The output of this system will be provided to the management personnel that will decide on how to approach the presence of an early fault in one of the idlers.

The method is good for systems that require a limited number of sensors and in contrast to the model-based method, it's easier to use in systems that have a nonlinear behavior (which sometimes is very hard to model/estimate). Also, it can be noticed that the input for this fault diagnosis system in represented by only a signal (acceleration), while for the model-based approach, several input signals were employed. Additionally, this type of fault diagnosis is strongly dependent on the sensors output, so if there would be a fault in the sensors, the system would probably signalize false faults.

Going further, there is a method with which also sensors' health is monitored.

\section{Fault Diagnosis for Sensors and Data Acquisition Systems}

In [11], Ding et al. present an advanced way of monitoring sensors that play a significant role in 3 vehicle systems: ABS (anti-lock breaking system), ESP (electronic stability program) and TCS (traction control system). The fault diagnosis system uses a model-based method, in which the sensors models are developed and 3 observers are used, each one generating two residual signals. In this work, the observers are built based on three time-discrete sensor models, which are suited to the vehicle dynamic system. The first residual signals are used for estimating model uncertainties and they are called Control Signals. The latter residual signals are called Detection Signals and are used for indicating the sensor faults.

This method shows the advantages that a modelbased fault diagnosis system can provide if the model is thoroughly developed and can detect sensor faults and even estimate the faulty sensor signal. For this type of systems, which other fault detection systems rely on, this is the best way to ensure a health monitoring, given the fact that if a signal is not correct, the entire system control can go wrong.

In [12], Jan et al. describe an intelligent method of monitoring the sensors using Support Vector Machines to classify faults that can appear in a temperature-to-voltage converter. They identify five faults that can appear in the sensor and train the system to classify the data into one of these faults or into a healthy system. Comparing this algorithm with the previous one, one can state that the latter is easier to implement, the hardest part being the training of the classifier. On the other hand, this system can only tell if there is a fault in the sensor, not being able to estimate a correct sensor signal and 
it's more prone to error than the model-based one. Of course, based on the type of sensor, each method has its benefits or downfalls, but based on the type of information that the sensor offers (life-critical data or just system data) the model-based system should be used over the artificial intelligence.

Since all the important subsystems that are part of a mechatronics system were covered, further it will be presented and discussed a fault diagnosis approach that tries to monitor the health of the entire system.

\section{Fault Diagnosis for a Complex System (Robot)}

Firstly, it is a look into a stochastic model-based method proposed by Mendoza et al. in [13], where they use the healthy robot model to assess whether there is a fault or not in a mobile robot. The method relies on three redundant information sources that can be used by an inference system. However, this article only presents a high level approach of a more complex task of identifying and detecting faults in a very complex system.

Another fault diagnosis approach is proposed by Liu and Coghill in [14], where a complex system is monitored using a model-based technique. This online robotic fault diagnosis approach is called "First Priority Diagnostic Engine" (FPDE). The proposed method presents a health monitoring system that is seen as a separated package from the robot itself and which detects sensor faults, model faults and robotic faults based on a first-priority parameter that is chosen for the robot and which is considered the principal parameter to be monitored.

The authors explain what robotic faults are. They can be described as orientation faults or translational faults, which can be caused by different components that are faulty. The system has an underlying system which is called "Componentbased robotic reasoning", which narrows down the fault at component level. They also present the reasoning algorithm, which is based on the interval filter (which is a system that extracts characteristic values of robot parameters), and which can actually categorize the faults "into behavior faults and modeling faults, based on sensor fault detection".

There are two other underlying systems: one is the bounds generator, which creates different bounds based on the robotic model for the different variables that are monitored; the other system is the fault reaction which decides what actions the robot should take, based on the discovered faults. This approach encapsulates artificial intelligence (interval filter) and model-based fault diagnosis and it can also be used for various mechatronics systems, while it's very useful for checking a system's model as well.

However, there are faults that can be diagnosed earlier, based on vibration patterns, before a certain monitored parameter goes out of bounds (for example the manipulator's trajectory).

In [15] An and et al. propose a hybrid intelligent approach for diagnosing a robot's manipulator. The authors use neural networks to model the robot's model nonlinearities and then use fuzzy logic to make an inference for detecting there is a fault or not in the robot's dynamics. This system also proposes a fault tolerance capability, which would allow the robot to work even if it's faulty. This way, the downtime is reduced and although there are faults in the robot, the production is not stopped. This article is very interesting as it captures very well the problems that have to be solved by a robust fault diagnosis system and it clearly states that having artificial intelligence algorithms makes the task much easier for online monitoring in complex systems.

Figure 10 is the scheme that describes quite accurately a predictive maintenance system for a complex system on a high level, according to the above presented algorithm.

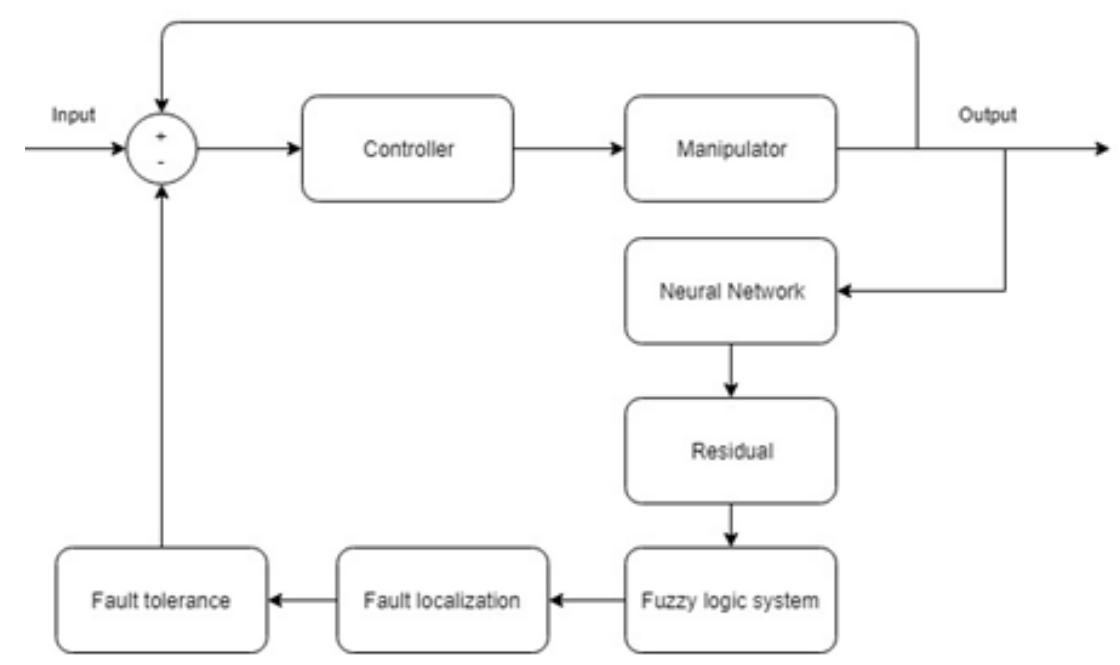

Figure 10: Diagram for a fault diagnosis system for a robot [15] 


\section{Future Work}

Starting from the concepts defined in the above presented works, a fault diagnosis system for a discrete model of a mechatronics system, that is generated by an artificial intelligence system will be developed.

This one could be used for a general system, that is treated as a combination of damped oscillators.

\section{References}

[1] Alexandros Mouzakitis, "Classification of Fault Diagnosis Methods for Control Systems", Measurement and Control, Vol. 46 (2013), Issue 10, 303-308

[2] Vandi Verma, Geoff Gordon, Reid Simmons, Sebastian Thrun, "Real-Time Fault Diagno-sis", IEEE Robotics \& Automation Magazine, June 2004

[3] V. M. Catterson, S. D. J. McArthur, "The Industrialization of a Multi-Agent System for Power Transformer Condition Monitoring", Applications and Innovations in Intelligent Systems XII, 2002, 165-178

[4] Rolf Isermann, "Model-based fault-detection and diagnosis - status and applications", Annual Reviews in Control 29 (2005) 71-85

[5] Hyun Cheol Cho, Kwang Su Kim, Chang Hwan Song, Young Jin Lee, Kwon Soon Lee, "Online Fault Detection and Diagnosis Algorithm based on Probabilistic Model for Induction Machines", SICE Annual Conference 2008

[6] Hua Su, Kil To Chong, "Induction Machine Condition Monitoring Using Neural Network Modelling", IEEE TRANSACTIONS ON INDUSTRIAL ELECTRONICS, Vol. 54, No. 1, February 2007
[7] Hua Su, Wang Xi, Kil To Chong, "Vibration Signal Analysis for Electrical Fault Detection of Induction Machine using Neural Networks", International Symposium on Information Technology Convergence, 2007

[8] Wenyi Wang, Albert K. Wong, "Autoregressive Model-Based Gear Fault Diagnosis", Journal of Vibration and Acoustics, April 2002, Volume $124 / 173$

[9] S. K. Ghoshal, S. Samanta, "Model Based Fault Diagnosis of a Belt Conveyor through Pa-rameter Estimation"

[10] Wei Li, Zewen Wang, Zhencai Zhu, Gongbo Zhou, Guoan Chen, "Design of Online Moni-toring and Fault Diagnosis System for Belt Conveyors Based on Wavelet Packet Decom-position and Support Vector Machines"

[11] S. X. Ding, S. Schneider, E. L. Ding, A. Rehm, "Advanced Model-Based Diagnosis of Sensors Faults in Vehicle Dynamics Control Systems", 16th Triennial World Congress, Prague, Czech Republic, 2005

[12] Sana Ullah Jan, Young-Doo Lee, Jungpil Shin, Insoo Koo, "Sensor Fault Classification Based on Support Vector Machine and Statistical TimeDomain Features", IEEE Access, May 2017

[13] Juan Pablo Mendoza, Manuela Veloso, Reid Simmons, "Mobile Robot Fault Diagnosis based on Redundant Information Statistics"

[14] Honghai Liu, George M. Coghill, "A modelbased approach to robot fault diagnosis", Knowledge-Based Systems 18 (2005) 225-233

[15] M. Dev. Anand, T. Selvaraj, S. Kumanan, "Fault Detection and Fault Tolerance Methods for Industrial Robot Manipulators based on Hybrid Approach", Advances in Production Engineering \& Management 7, 2012, 225-226 